\title{
GMR
}

\section{Identification of Colletotrichum isolates from Capsicum chinense in Amazon}

\author{
C.V.S. de Oliveira ${ }^{1}$, K.S. Matos ${ }^{2}$, D.M.C. de Albuquerque ${ }^{1}$, R.E. Hanada ${ }^{2}$ \\ and G.F. da Silva ${ }^{1}$ \\ ${ }^{1}$ Laboratório de Biologia Molecular, Embrapa Amazônia Ocidental, Manaus, \\ AM, Brasil \\ ${ }^{2}$ Instituto Nacional de Pesquisa da Amazônia, Manaus, AM, Brasil \\ Corresponding author: G.F. Silva \\ E-mail: gilvan.silva@embrapa.br \\ Genet. Mol. Res. 16 (2): gmr16029601 \\ Received January 10, 2017 \\ Accepted May 29, 2017 \\ Published June 29, 2017 \\ DOI http://dx.doi.org/10.4238/gmr16029601
}

Copyright $(2017$ The Authors. This is an open-access article distributed under the terms of the Creative Commons Attribution ShareAlike (CC BY-SA) 4.0 License.

\begin{abstract}
Chili pepper (Capsicum chinense) is a great economic important culture on the State of Amazonas, and it represents, approximately, a production of 1.9 thousand tons per year. It is one of the hosts of Colletotrichum genus in the North region of Brazil. The aim of the study was to differentiate and to identify isolates of Colletotrichum collected from C. chinense in Amazon. Molecular characterization, using RFLP-PCR, ERIC-PCR and ISSR, was carried out initially for screening of morphologically similar isolates. Furthermore, phylogenetic analyses were performed using combined regions: Actin (ACT), glyceraldehyde-3-phosphate dehydrogenase (GAPDH) for the three isolates, INPA 2066, INPA 2286 and INPA 1858, plus superoxide dismutase (SOD2) for INPA 2066. We showed that the molecular markers were able to distinguish the isolates of Colletotrichum studied and these results were confirmed with the phylogenetic analyses, three different occurrences of Colletotrichum species (C. siamense, C. scovillei and C. brevisporum) causing anthracnose in $C$. chinense in the State of Amazonas. This study
\end{abstract}

Genetics and Molecular Research 16 (2): gmr16029601 
represents the first report of the species C. siamense and C. scovillei in this host in Brazil.

Key words: Colletotrichum; Molecular markers; Chili pepper; Phylogenetic analysis

\section{INTRODUCTION}

Colletotrichum (teleomorph Glomerella), comprises a big range of cosmopolitan fungi species and are usually described as being the anthracnose disease agent. It is known $\sim 600$ species of the genus Colletotrichum, which are pathogens of over 3000 species of plants including cereals, legumes, vegetables, perenial crops, and tree fruits (O'Connell et al., 2012). There are some Colletotrichum species complexes already described in the literature, such as C. gloeosporioides, C. acutatum, C. boninense and C. orbiculare (Weir et al., 2012; Damm et al., 2012a,b, 2013). In Brazil, there are many reports of plant infection caused by Colletotrichum species, especially in economically important plants and fruits, such as coffee berries, sugarcane, strawberry, maize, sorghum, banana, avocado and many others. The tropical and subtropical climate favors the spread of Colletotrichum species on these plants.

It is notable the great economic interest of chili pepper (Capsicum chinense Jacq.) culture in many parts of the world. It happens because of its great potential of growth in almost every country, since it is a tropical regions native, and its use on food, pharmaceutical, cosmetic, and ornamental products (Dias et al., 2013). C. chinense is one of the most cultivated vegetables in Brazil, mainly in the North region. The anthracnose is a disease that more affects the culture and five species of Colletotrichum were described as the pathogen for this host, C. capsici and C. gloeosporioides in India, Indonesia, Korea, and Thailand, C. acutatum in Australia and Indonesia, C. coccodes in Korea and New Zealand (Ratanacherdchai et al., 2010) and C. brevisporum in Brazil (Almeida et al., 2017).

The identification of Colletotrichum based on morphological characters is problematic due to the few morphological traits that can be used to separate species in this genus (Than et al., 2008). Therefore, it is necessary a precise study and characterization based not only on morphological but also on molecular data utilized for species delimitation and defining of inter- and intraspecific relationships as it has been performed in the past decades. Several molecular techniques have been developed to characterize and to identify different Colletotrichum species. Multilocus phylogenetic analysis using partial sequences of gene such as actin (ACT), calmodulin (CAL), chitin synthase (CHS-1), glyceraldehyde-3phosphate dehydrogenase (GAPDH), glutamine synthetase (GS), and manganese superoxide dismutase (SOD2) have been utilized to identify different species of this genus (Weir et al., 2012; Damm et al., 2012a).

Additionally, molecular markers are generally recognized as a reliable method to evaluate genetic diversity and differentiation of Colletotrichum spp isolates, such as restriction fragments length polymorphism (RFLP-PCR), inter-simple sequence repeats or microsatellites (ISSR) and enterobacterial repetitive intergenic consensus (ERIC-PCR). In the present paper, the objective was to differentiate and identify isolates of Colletotrichum collected from $C$. chinense in Amazon, northern of Brazil, using molecular markers and phylogenetic analysis.

Genetics and Molecular Research 16 (2): gmr16029601 


\section{MATERIAL AND METHODS}

\section{Isolation of Colletotrichum from C. chinense}

Fruits of chili pepper (C. chinense Jacq.) with typical anthracnose symptoms were observed in Manaus, Amazonas, Brazil.

The isolation was performed by collecting spores directly from the surface of the lesions in C. chinense fruit and then plated on PDA culture medium. A monosporic culture was performed to ensure that this work would be upon a single genetic uniformity.

The isolates of Colletotrichum selected and used for screening analysis (Figures 1, 2 and 3) and phylogenetic study are deposited in the culture collection of the Phytopathology Laboratory of National Institute of Amazonian Research - INPA (INPA 2286, INPA 2066 and INPA 1858) (Table 1).

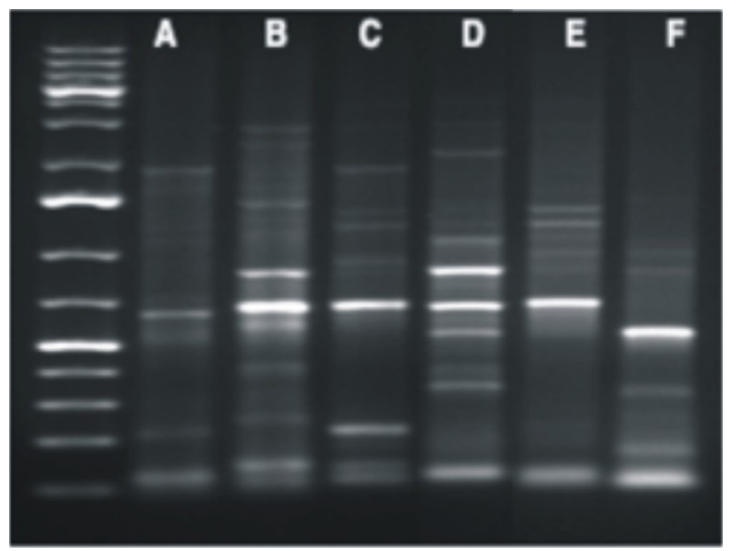

Figure 1. ERIC-PCR of the three isolates from Capsicum chinense. A. Colletorihcum fructicola. B. C. gloeosporioides. C. C. fragariae. D. Isolate 2286. E. Isolate 2066. F. Isolate 1858.

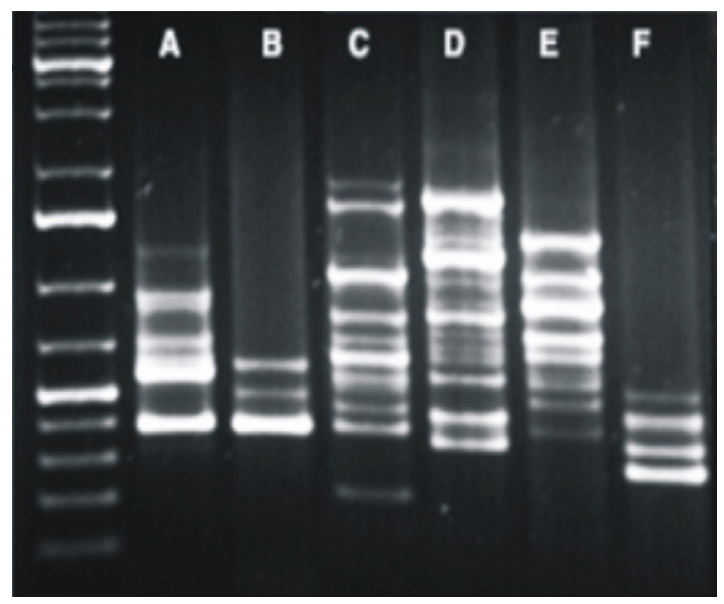

Figure 2. PCR amplification of ISSR of the three isolates from Capsicum chinense. A. Isolate 1858. B. Isolate 2066. C. Isolate 2286. D. Colletorihcum fructicola. E. C. fragariae. F. C. gloeosporioides. 


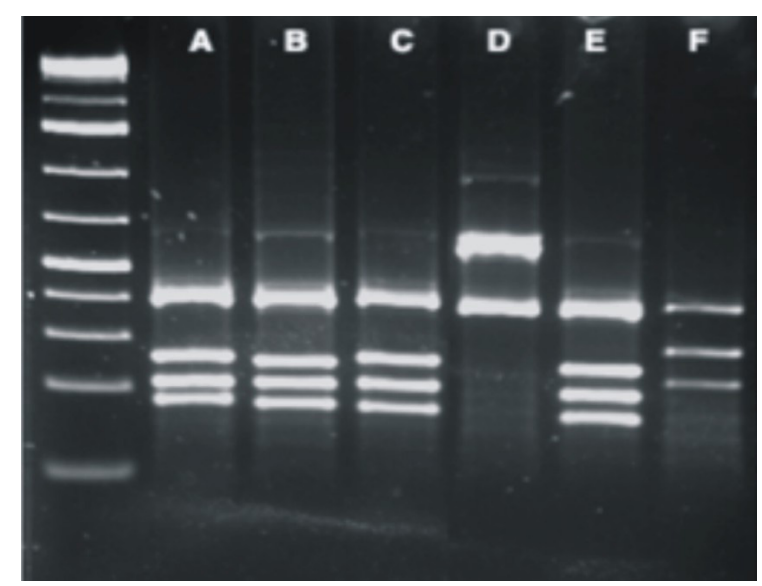

Figure 3. PCR amplification of the 1-kb GS intron based on Pst enzyme digestion (RFLP-PCR), of the three isolates from Capsicum chinense. A. Colletorihcum fructicola. B. C. gloeosporioides. C. C. fragariae. D. Isolate 2286. E. Isolate 1858. F. Isolate 2286.

Table 1. List of primers used for molecular screening and phylogenetic analysis.

\begin{tabular}{l|l|l}
\hline Region & Primers & Sequences (5'-3') \\
\hline Enterobacterial Repetitive Intergenic Consensus & ERIC1 & ATGTTAAGTCCCTGGGGATTCAC \\
\hline Enterobacterial Repetitive Intergenic Consensus & ERIC2 & AGTAAGTGACTGGGGTGAGCG \\
\hline Glutamine synthetase & GSF1 & ATGGCCGAGTACATCTGG \\
\hline Glutamine synthetase & GSR1 & AACCGTCGAAGTTCCAC \\
\hline Inter Simple Sequence Repeats & UBC 885 & BHBGAGAGAGAGAGAGA \\
\hline Actin & ACT 512 F & ATGTGCAAGGCCGGTTCGC \\
\hline Actin & ACT 783 R & TACGAGTCCTTCTGGCCCAT \\
\hline Superoxide dismutase & SODglo2-F & CAG ATC ATG GAG CTG CAC CA \\
\hline Superoxide dismutase & SODglo2-R & TAG TAC GCG TGC TCG GAC AT \\
\hline Glyceraldehyde 3-Phosphate dehydrogenase & GAPDH-F & GCCGTCAACGACCCCTTCATTGA \\
\hline Glyceraldehyde 3-phosphate dehydrogenase & GAPDH-R & GGGTGGAGTCGTACTTGAGCATGT \\
\hline
\end{tabular}

\section{Screening Colletotrichum isolates by molecular markers}

To confirm if $C$. chinense, in the State of Amazonas, is host of different species of Colletotrichum, a initial screening on the obtained isolates was carried out only using different molecular profile isolates for phylogenetic analysis. For this screening, we used ERIC-PCR, ISSR and PCR-RFLP of GS (Glutamine sintase) intron techniques. For comparison, we used isolates of reference of C. fructicola, C. gloeosporioides and C. fragariae.

DNA extraction was carried out at the Molecular Biology Laboratory - Embrapa Western Amazon, according to Doyle and Doyle (1987). The primers used for amplification are listed in Table 2.

To ERIC-PCR marker, ERIC1 and ERIC2 primers were used at a concentration of 0.2 $\mu \mathrm{M}$; 1X Buffer [100 mM Tris-HCl pH 8.8; $500 \mathrm{mM} \mathrm{KCl,} \mathrm{0.8 \%} \mathrm{(v/v)],} 25 \mathrm{mM} \mathrm{MgCl}_{2} ; 0.5 \mathrm{mM}$ dNTPs; 50 ng DNA; 1 U Taq polymerase (DNA Express ${ }^{\mathbb{B}}$ ), and reaction was set up to a final volume of $25 \mu \mathrm{L}$. The programming on thermal cycler (Applied Biosystems Veriti ${ }^{\mathrm{TM}}$ 96-Well Thermal Cycler) initiated with $94^{\circ} \mathrm{C}$ for 1 min followed by 35 cycles of denaturation $\left(94^{\circ} \mathrm{C}\right.$ for $1 \mathrm{~min})$, annealing $\left(48^{\circ} \mathrm{C}\right.$ for $\left.1 \mathrm{~min}\right)$ and elongation $\left(65^{\circ} \mathrm{C}\right.$ for $\left.5 \mathrm{~min}\right)$ and further extension of $65^{\circ} \mathrm{C}$ for $6 \mathrm{~min}$. 
Table 2. Strains of Colletotrichum spp used for phylogenetic analysis, with collection details and GenBank accessions.

\begin{tabular}{|c|c|c|c|c|c|c|}
\hline \multirow{2}{*}{$\begin{array}{l}\text { Species names } \\
\text { C. aenigma }\end{array}$} & \multirow{2}{*}{\begin{tabular}{|l|} 
Culture" \\
\end{tabular}} & Host/Substrate & \multirow{2}{*}{ Origin } & \multicolumn{3}{|c|}{ GenBank accession No, } \\
\hline & & Persea americana & & 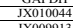 & $\begin{array}{l}\text { JX009443 } \\
\text { J09991 }\end{array}$ & 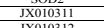 \\
\hline C. aeschynomenes & 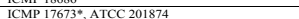 & $\begin{array}{l}\text { Pyrus pyryolat } \\
\text { Aeschyome viryinica }\end{array}$ & & IX009930 & $\begin{array}{l}J \times 00919 \\
J 009483\end{array}$ & \\
\hline C. alienum & ICMP 12071" & Malus domestica & New Zeland & JX010028 & JX009572 & JX010333 \\
\hline & ICMP 18621 & Persea americana & New Zeland & JX009959 & JX009952 & JX010308 \\
\hline C. communis (C. cf. siamense) & & Bauhinia variegata & & KC790736 & KC790622 & - \\
\hline C. diamesei (syn.C. melanocaulon) & $\begin{array}{l}\text { GS06 } \\
\text { CMM } 4083 \\
\end{array}$ & Saracacandica & Bndiazil & KCF5171949 & $\begin{array}{l}\text { KCC90625 } \\
\text { KC517298 }\end{array}$ & $\therefore$ \\
\hline & 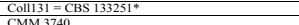 & Vaccinium macrocarpon & USA & $\begin{array}{l}\text { KP703275 } \\
K C 51709\end{array}$ & & - \\
\hline C. endomangfferae & $\begin{array}{l}\text { CMM } 3740 \\
\text { CMM 3814* }\end{array}$ & $\begin{array}{l}\text { Mangifiera itdica } \\
\text { Mangifera indica }\end{array}$ & $\begin{array}{ll}\text { Brazal } \\
\text { Brazil }\end{array}$ & $\frac{K C S 17298}{K C 517298}$ & $\begin{array}{l}\text { KC5517298 } \\
\text { KC517298 }\end{array}$ & - \\
\hline C. fruticola & ICMP $18581^{\circ}$, CBS 130416 & Colfea arabica & Thailand & JX010033 & FI907426 & JX010327 \\
\hline & ICMP 17921, CBS 238.49" & Ficus edululis & Germany & JX009923 & JX009495 & JX010322 \\
\hline C. hebeiense & $\frac{S D 452}{K 3}$ & 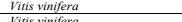 & China & KF377505 & $\frac{\mathrm{KF} 377542}{\mathrm{KE} 377532}$ & - \\
\hline C. hymenocallidis (C. cf. siamense) & $\operatorname{CSSN}^{*}$ & Hymenocallis americana & China & & GQ856775 & \\
\hline C. murrayae (C. cof siamense) & $\begin{array}{l}\text { CSAN3 } \\
\text { GZAAS5.09538 }\end{array}$ & $\begin{array}{l}\text { Hymenocallis americana } \\
\text { Murrava sp }\end{array}$ & $\frac{\text { China }}{\text { China }}$ & .0277608 & $\begin{array}{l}\text { GQ856776 } \\
1024756\end{array}$ & - \\
\hline & GZAAS5.09506 & & China & JQ247609 & JQ247657 & \\
\hline C. musae & 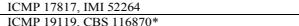 & $\begin{array}{l}\text { Musa sapientum } \\
\text { Musaspents }\end{array}$ & $\begin{array}{ll}\text { Kenya } \\
\text { UISI }\end{array}$ & $\begin{array}{lll}\text { IX010015 } \\
\times 001000\end{array}$ & $\begin{array}{l}J \times 009432 \\
J \times 09433 \\
\end{array}$ & JX010317 \\
\hline C. mupharicola & $\begin{array}{l}\text { CBS 469.96, IMCP } 17938 \\
C^{2}\end{array}$ & 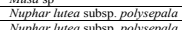 & $\begin{array}{ll}\text { USA } \\
\text { USA }\end{array}$ & 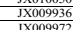 & 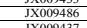 & 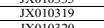 \\
\hline C. queensalandicum & ICMP 1778* & 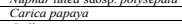 & Australia & JX009934 & JX009447 & $\begin{array}{l}\text { JX01020 } \\
\text { JX0136 }\end{array}$ \\
\hline & ICMP 18705 & & & JXX10036 & JX009490 & \\
\hline $\begin{array}{l}\text { C. salasolae } \\
\text { C.simense }\end{array}$ & ICMP $19057^{\circ *}$ & $\begin{array}{l}\text { Salsoa traguss } \\
\text { Coféarabica }\end{array}$ & Hungary & JX009916 & JX009562 & JX010325 \\
\hline C. Silamense & INPA 2066 & $\begin{array}{l}\text { Collea arabica } \\
\text { Capsicum chinense }\end{array}$ & Amazonas, Brazil & KY & $\begin{array}{l}\mathrm{F} J 90 / 23 \\
\mathrm{KY} 335609\end{array}$ & $\begin{array}{l}\text { JXY101026 } \\
\text { KY43513 }\end{array}$ \\
\hline C. siamense (syn. C. hymenocallidis) & $\begin{array}{l}\text { ICMP 18642, CBS } 125377^{*} \\
\end{array}$ & Hymenocallidis americana & & JX010019 & GQ856775 & $\begin{array}{l}\mathrm{J} \times 010332 \\
\end{array}$ \\
\hline C.tropicale & $\begin{array}{l}\text { ICMP } 18672, \text { MAFF } 239933 \\
\text { ICMP 18653, CBS } 124949^{*}\end{array}$ & $\begin{array}{l}\text { Litchl chinensis } \\
\text { Theobroma cacao } \\
\end{array}$ & $\begin{array}{l}\frac{J_{\text {apan }}}{\text { Panama }} \\
\end{array}$ & $\begin{array}{l}\text { IX0100020 } \\
\text { IX010007 }\end{array}$ & $\begin{array}{l}\text { JX009480 } \\
\text { JX009489 } \\
\end{array}$ & $\begin{array}{l}\text { JX010318 } \\
\text { JX010329 } \\
\end{array}$ \\
\hline $\begin{array}{l}\text { C. acerbum } \\
\text { Cocuturyun }\end{array}$ & 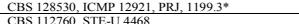 & Malus domestica & New Zeland & $\begin{array}{l}\mathrm{J} \text { JQ948790 } \\
\mathrm{J}\end{array}$ & JQ949780 & - \\
\hline C. acutatum & $\begin{array}{l}\text { CBS } 1112760, \text { STEEU } 4468 \\
\text { CBS } 112761 \text {, STE-U } 4461\end{array}$ & $\begin{array}{l}\text { Hakea sericea } \\
\text { Hakea sericea }\end{array}$ & $\begin{array}{l}\frac{S}{\text { South Africa }} \\
\text { South Africa }\end{array}$ & $\begin{array}{l}\text { JQ948723 } \\
\text { JQ948724 }\end{array}$ & 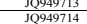 & $\therefore$ \\
\hline & CBS 113599, STE-U 3038 & Grevillea sp & Australia & JQ948678 & JQ949668 & \\
\hline C. australe & CBS 116478, HKUCC 2616" & $\begin{array}{l}\text { Trachycarpus fortune } \\
\text { The }\end{array}$ & South Africa & IQ948786 & JQ949711 & - \\
\hline & $\begin{array}{l}\text { CBS } 131325, \text { CPC } 19820 \\
\end{array}$ & Hakea sp. & Australia & JQ948787 & JQ949777 & \\
\hline $\begin{array}{l}\text { C. brisbonense } \\
\text { C. chrysanthemi }\end{array}$ & \begin{tabular}{|l|l|} 
IMI 3645450, , CPC 18930 \\
\end{tabular} & $\begin{array}{l}\text { Capsicum anmumum } \\
\text { Chrysanthemum coronarium }\end{array}$ & \begin{tabular}{|l} 
Australia \\
China \\
\end{tabular} & 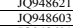 & $\begin{array}{l}\frac{\mathrm{J} Q 999612}{\mathrm{JQ} 949594} \\
\end{array}$ & $\therefore$ \\
\hline C. cosmi & 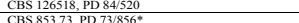 & $\begin{array}{l}\text { Carthamus sp } \\
\text { Commos }\end{array}$ & $\begin{array}{l}\text { Netherlands } \\
\text { Notherlands }\end{array}$ & $\begin{array}{l}\text { JQ948601 } \\
\text { JO946604 }\end{array}$ & $\begin{array}{ll}\text { JQ949592 } \\
\text { J0449955 }\end{array}$ & - \\
\hline C. costaricense & CBS 330.75" & Coffea arabica & Costa Rica & Jo948510 & JQ949501 & \\
\hline C. cuscutae & \begin{tabular}{|l|l|} 
CBI 211.178, IMI \\
IMI 30402 CPC 18873*
\end{tabular} & $\begin{array}{ll}\text { Coffea } \mathrm{sp} \\
\text { Cosutasp }\end{array}$ & $\begin{array}{l}\text { Costa Rica } \\
\text { Dominical }\end{array}$ & $\begin{array}{l}\text { JQ948511 } \\
\text { J094525 }\end{array}$ & $\begin{array}{l}0949502 \\
099516\end{array}$ & - \\
\hline C. fioriniae & CBS 129946, PT170, RB021 & Olea europaea & Portugal & JQ948672 & JQ94996663 & \\
\hline & CSL318 & Magnolia sp & UK & Jo948676 & JQ949667 & - \\
\hline C. godetiae & IMI 381927, CPC 18935 & Rubus idaenus & Turkey & JQ948769 & JQ949759 & - \\
\hline C. guajiavae & 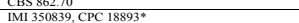 & $\begin{array}{l}\text { Sambucus nigra } \\
\text { Psidium guajava }\end{array}$ & $\begin{array}{l}\text { Nefterlainas } \\
\text { India }\end{array}$ & JQ99486600 & $\frac{1049958}{J 094991}$ & \\
\hline$C$, indonesiense & CBS 127551, CPC $14986^{*}$ & Eucalyptus sp & Indonesia & JQ948618 & JQ949609 & \\
\hline C. jofhsilonit & CBS 128532, ICMP 12926, PRJ 1139.3* & Solanum lycopersicum & New Zeland & JOQ948775 & JQ949765 & \\
\hline & CBS $198.35^{\circ}$ & Phormium sp & UK & JQ948785 & JQ949775 & - \\
\hline C. laticiphilum & $\begin{array}{l}\text { CBS 112989, IMI } 383015 \text {, STE-U 5303* } \\
\text { CBS 129827 CH2 }\end{array}$ & $\begin{array}{l}\text { Hevea brasiliensis } \\
\text { Hevea brasiliensis }\end{array}$ & India & $\frac{\text { JQ944619 }}{\text { JQ948620 }}$ & $\begin{array}{l}\text { JQ949610 } \\
\text { J0949611 }\end{array}$ & $\overline{-}$ \\
\hline C.limeticola & CBS 114.14* & Citrus aurantifolia & USA, Florida & 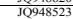 & J0949514 & \\
\hline C. melonis & \begin{tabular}{|l|l|} 
CBS1 19142, CMW 9931 \\
CBS 15984*
\end{tabular} & $\begin{array}{ll}\text { Lupinus albus } \\
\text { Curcunis melo }\end{array}$ & South Africa & $\begin{array}{ll}\text { JQ948505 } \\
\end{array}$ & JQ949496 & - \\
\hline C. nymphaeae & CBS 129933, Goffly9 & $\begin{array}{c}\text { Cucums melo } \\
\text { Fragaria } x \text { ananassa }\end{array}$ & USA & & & \\
\hline & IMI 348177, CPC $18890^{\circ}$ & Fragaria $\times$ ananassa & $\begin{array}{lll}\text { USA } & \\
\end{array}$ & JQ948593 & JQ949584 & - \\
\hline C. paxtonii & $\begin{array}{ll}\text { CBS 502.97, LARS } 58 \\
\end{array}$ & Musa nana & "West Indies" & J0948616 & JQ949607 & - \\
\hline C. phormii & CBS 118197, AR 3389 & $\begin{array}{lll}\text { Mussasp } \\
\text { Phorviun sp }\end{array}$ & $\begin{array}{l}\text { Salmt Lucla } \\
\text { New Zeland }\end{array}$ & 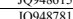 & $\begin{array}{l}\text { JQ9496606 } \\
\text { J0949771 }\end{array}$ & \\
\hline & CBS 483.82 & $\begin{array}{l}\text { Phormuming } \mathrm{p} \text { tenax } \\
\text { Phoris }\end{array}$ & New Zeland & 10948782 & 10949772 & $-\pi$ \\
\hline & CBS 118194, AR $3546^{*}$ & Phormium sp & Germany & JQ948777 & JQ949767 & $\therefore$ \\
\hline C. pyricola & CBS 128531, ICMP 12924, PRJ 977.1* & Pyrus communis & New Zeland & JQ948776 & JQ949766 & \\
\hline C. rhombiforme & CBS 129953, PT250, RB011* & Olea europaea & Portugal & JQ948788 & JQ948778 & - \\
\hline & CBS 131322, DAOM 233253, Cl0, MSIL34 & Vaccinium macrocarpum & USA & JO948789 & JQ949779 & 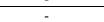 \\
\hline C. salicis & $\begin{array}{ll}\text { IMI } 345585, \text { CPC } 19376 \\
\end{array}$ & Fragaria $\mathrm{x}$ ananassa & New Zeland & J0948807 & J0949797 & - \\
\hline & CBS 239.49 & Unknown & $\begin{array}{lll}\text { Unknown } \\
\end{array}$ & JQ948800 & JQ949790 & - \\
\hline C. scovillei & $\begin{array}{l}\text { CBS 126529, PD 94/924-3, BBA70349* } \\
\end{array}$ & Capsicum sp & Indonesia & JQ948597 & JQ949588 & - \\
\hline & & Capsicum sp & Indonesia & JQ948598 & JQ949589 & \\
\hline & CBS 120708 & Capsicum annuयm & Thailand & 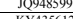 & JQ949590 & - \\
\hline C. scovillei & INPA 2286 & $\begin{array}{l}\text { Capsicum chinense } \\
\text { Protegcyopider }\end{array}$ & Amazonas, Brazil & KY435612 & KY435610 & - \\
\hline & 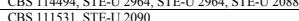 & $\begin{array}{l}\text { Protea cynarondes } \\
\text { Protec cynaroides }\end{array}$ & USA & 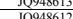 & $\frac{1049964}{10949603}$ & $\overline{-}$ \\
\hline C. solanei & IMI 364297, CPC 18929** & Theobroma cacao & Malaysia & J0948617 & JQ949608 & - \\
\hline C. tamarilloi & CBS 12814, T.A.6* & Solanum betaceum & Colombia & JQ948514 & JQ949505 & - \\
\hline & CBS 129811, T.A.3 & Solanum betaceum & Colombia & JQ948 & JQ949506 & - \\
\hline $\begin{array}{l}\text { C. walerii } \\
\text { Corbiculare }\end{array}$ & $\begin{array}{ll}\text { CBS } 125472, \text { BMT (HL) } 19^{*} \\
\end{array}$ & Coffea sp & Vietnam & JQ948605 & JQ949596 & - \\
\hline $\begin{array}{l}\text { C. orbriculare } \\
\text { C. brevisporum }\end{array}$ & $\begin{array}{l}\text { CBS 133198, KTU-K6 } \\
\text { L } 57 \text { LC6000 BCC } 38876^{*}\end{array}$ & $\frac{\text { Cucumis melo }}{\text { Neoregalia sp }}$ & 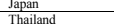 & 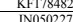 & $\frac{\mathrm{KFF} 78555}{\mathrm{JN} 050216}$ & \\
\hline 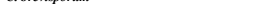 & BTL23, LC0870, MFLUCC 100182 & Pandanus pyymaeus & Thailand & JN050228 & JN050217 & . \\
\hline & INPA 1858 & Capsicum chinense & Amazonas, Brazil & KX878887 & KX878886 & - \\
\hline C. boninense & $\begin{array}{l}\text { CBS123755, MAFF 305972* } \\
\end{array}$ & Crinum asiaticum var. sinicum & Japan & JQ005240 & JQ005501 & - \\
\hline C. cliviae & CSSSI & Clivia miniata & China & GU085867 & GU08586l & - \\
\hline & CSSS2 & Clivia miniata & China & GU085868 & GU085862 & - \\
\hline $\begin{array}{l}\text { C. kahawae subsp. rigge } \\
\text { (syn.Glomerella rufoma }\end{array}$ & CBS 124.22*, ICMP 19122 & Vaccinium sp & USA & JX009950 & JX009536 & - \\
\hline C. karstii & CBS 129833 & Musa sp & Mexico & JQ005262 & JQ005 & - \\
\hline C. thailandicum & \begin{tabular}{|l} 
HR01MFU, LC \\
\end{tabular} & Hibiscus rosa-sinensis & Thailand & JN050 & JN050220 & - \\
\hline & CMSP34, LC0958, MFUCC 100192 & Alocasia sp & Thailand & JN050232 & JN050221 & - \\
\hline C. trifollii & CBS 128554, ICMP 12934, & Medicago sativa & & KF178500 & KF1785 & \\
\hline C. tropicicola & 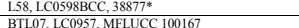 & $\frac{\text { Citrus maxima }}{\text { Paphiopedilum bellatolum }}$ & \begin{tabular}{|l|l|l|l|l} 
Thailand \\
Thailand
\end{tabular} & $\frac{J \mathrm{~N} 050229}{\mathrm{JNN} 050230}$ & $\begin{array}{l}\text { JN050218 } \\
\text { JN500219 }\end{array}$ & $\therefore$ \\
\hline
\end{tabular}

aTCC:American Type Culture Collection, Virginia, USA; BBA: Culture collection of the Biologische Bundesanstalt für Land- und Forstwirtschaft, Berlin, Germany; BRIP: Plant Pathology Herbarium, Department of Primary Industries, Queensland, Australia; CBS: Culture collection of the Centraalbureau voor Schimmelcultures, Fungal Biodiversity Centre, Utrecht, The Netherlands; CPC: Culture collection of Pedro Crous, housed at CBS; CMM: Coleção de Culturas de Fungos Fitopatogênicos "Prof.Maria Menezes", Federal University of Pernambuco, Recife, Brazil; DAOM: Plant Research Institute, Department of Agriculture (Mycology), Ottawa, Canada; IMI: Culture collection of CABI Europe UK Centre, Egham, UK; LARS: Culture collection of Long Ashton Research Station, Bristol, UK (no longer existing); MAFF: MAFF Genebank Project, Ministry of Agriculture, Forestry and Fisheries, Tsukuba, Japan; ICMP: International Collection of Microorganisms from Plants, Auckland, New Zealand; MFLUCC: Mae Fah Luang University Culture Collection, ChiangRai, Thailand; STE-U: Culture collection of the Department of Plant Pathology, University of Stellenbosch, South Africa; INPA: National Institute of Amazonian Research Manaus, Amazonas, Brazil; HKUCC: The University of Hong Kong Culture Collection, Hong Kong, China; PD: Plantenziektenkundige Dienst Wageningen, Nederland. 'blyceraldehyde3-phosphate dehydrogenase (GAPDH), Actin (ACT) and Manganese superoxide dismutase (SOD2) were sequences used in the phylogenetic analyses. The Colletotrichum strains used in this study are indicated in bold. *Ex-holotype or ex-epitype cultures.

Genetics and Molecular Research 16 (2): gmr16029601 
To ISSR marker analysis, the PCR was performed at a $25-\mu \mathrm{L}$ final reaction volume, with $0.2 \mu \mathrm{M}$ of the UBS primer 885; 1X Buffer [100 mM Tris-HCl pH 8.8; $500 \mathrm{mM} \mathrm{KCl,} 0.8 \%$ (v/v)]; $25 \mathrm{mM} \mathrm{MgCl}_{2} ; 0.5 \mathrm{mM}$ dNTPs; $50 \mathrm{ng}$ DNA; $1.5 \mathrm{U}$ Taq Polymerase (DNA Express ${ }^{\circledR}$ ). The pre-cycle was on $94^{\circ} \mathrm{C}$ for $5 \mathrm{~min}$, followed by 40 cycles of denaturation $\left(94^{\circ} \mathrm{C}\right.$ for $\left.1 \mathrm{~min}\right)$, annealing $\left(45^{\circ} \mathrm{C}\right.$ for $\left.1 \mathrm{~min}\right)$ and elongation $\left(72^{\circ} \mathrm{C}\right.$ for $\left.1 \mathrm{~min}\right)$, followed by a final extension at $72^{\circ} \mathrm{C}$ for $7 \mathrm{~min}$. Amplification of intron GS, PCR-RFLP was carried out according to Liu et al. (2012). All PCR and PCR-RFLP products ran at a 1.5\% w/v agarose gel electrophoresis.

\section{Phylogenetic analysis}

Three loci were amplified by PCR and we used for phylogenetic analysis: ACT, GAPDH and SOD2. For these reactions, 1X Buffer [(100 mM Tris-HCl pH 8.8; $500 \mathrm{mM}$ $\mathrm{KCl}, 0.8 \%(\mathrm{v} / \mathrm{v})], 25 \mathrm{mM} \mathrm{MgCl}_{2} ; 10 \mathrm{mM}$ dNTPs; $5 \mu \mathrm{M}$ for each primer; $50 \mathrm{ng} / \mathrm{mL}$ DNA; 5 U Taq Polymerase (DNA Express ${ }^{\circledR}$ ). The first cycle initiated with $94^{\circ} \mathrm{C}$ for $4 \mathrm{~min}$, followed by 35 cycles of denaturation $\left(94^{\circ} \mathrm{C}\right.$ for $\left.30 \mathrm{~s}\right)$, annealing $\left(60^{\circ} \mathrm{C}\right.$ for $\left.30 \mathrm{~s}\right)$ and elongation $\left(72^{\circ} \mathrm{C}\right.$ for $1 \mathrm{~min}$ ), followed by a final extension at $72^{\circ} \mathrm{C}$ for $7 \mathrm{~min}$. The primer sequences used for each gene are described in the Table 2. PCR products were purified and sequenced by the Applied Biosystems ${ }^{\circledR} 3500$ Genetic Analyzers. Sequences from forward and reverse primers were aligned to obtain a consensus sequence (Table 1). The fungal DNA sequences were aligned using MEGA 6 (Tamura et al., 2013) with reference sequences of the Colletotrichum obtained from GenBank. The Bayesian inference analyses employing a Markov Chain Monte Carlo method were performed with all individual and combined sequences. The MrModeltest 2.3 (Posada and Buckley, 2004) was used to determine the best model of nucleotide evolution $(\mathrm{HKY}+\mathrm{I}$ to ACT; HKY+G to GAPDH; GTR $+\mathrm{I}+\mathrm{G}$ to SOD2). The phylogenetic analysis was performed on CIPRES web portal (Miller et al., 2010) using MrBayes version 3.2 (Ronquist et al., 2012). Markov chain Monte Carlo method was run for 10,000,000 generations, sampling every 1000 generations and discarding 2500 samples as burn-in. The resulting trees were rooted using outgroup taxon. Trees were visualized in FigTree 1.4.0 (Rambaut, 2012) and exported to graphic programs. The sequences obtained in this study were deposited in GenBank (Table 2).

\section{RESULTS AND DISCUSSION}

The results obtained reveal that simple molecular settings such as ERIC-PCR, PCRRFLP and ISSR can be used with efficiency for screening of Colletotrichum isolates aiming to identify different species capable of causing anthracnose in the same host. ERIC-PCR and ISSR unique band profiles can be identified (Figures 1 and 2). GS RFLP-PCR also evidenced different profile bands for the isolates (Figure 3).

Phylogenetic results revealed that these different profiles correspond to different species of Colletotrichum associated with $C$. chinense (Figures 4, 5 and 6). This information could be confirmed by Bayesian inference methods from multiple gene sequences.

Sequences from fragments of ACT, GAPDH and SOD2 from isolate INPA 2066, ACT and GAPDH from isolates INPA 2286, and INPA 1858 from C. chinense were compared with sequences from strains of other Colletotrichum species and it showed approximately $99 \%$ of similarity with Colletotrichum siamense, $99 \%$ of similarity with C. scovillei Damm, P.F. Cannon \& Crous, and 96\% of identity with C. brevisporum Phoulivong, P. Noireung, L. Cai \& K.D. Hyde, respectively.

Genetics and Molecular Research 16 (2): gmr16029601 


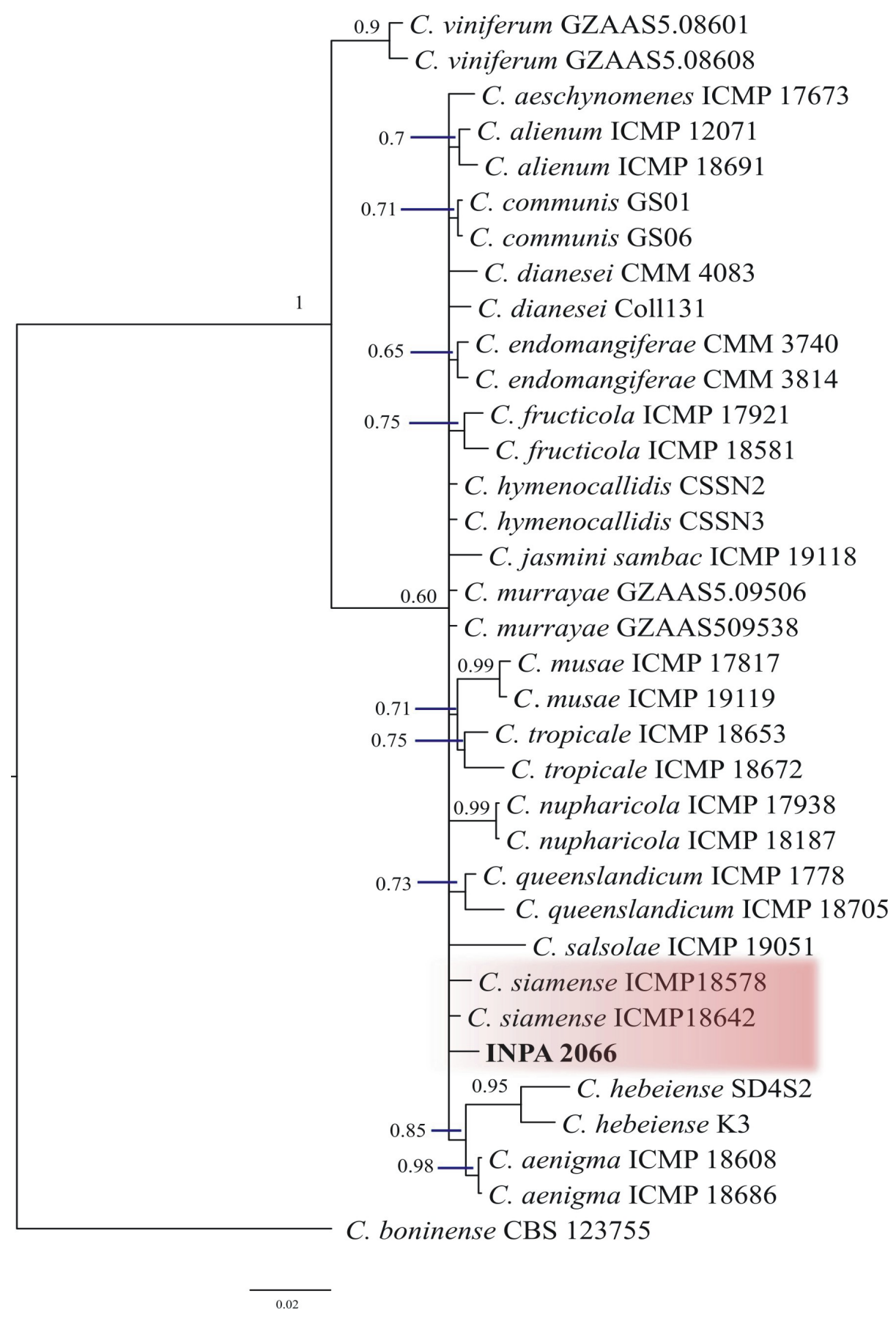

Figure 4. Phylogenetic tree generated by Bayesian inference upon a combinated ACT, GAPDH and SOD2 alignment sequences of Colletotrichum gloeosporioides species complex and the INPA 2066 isolate highlighted. This tree is rooted with $C$. boninense. Relevant bootstrap values are shown ate the nodes. 


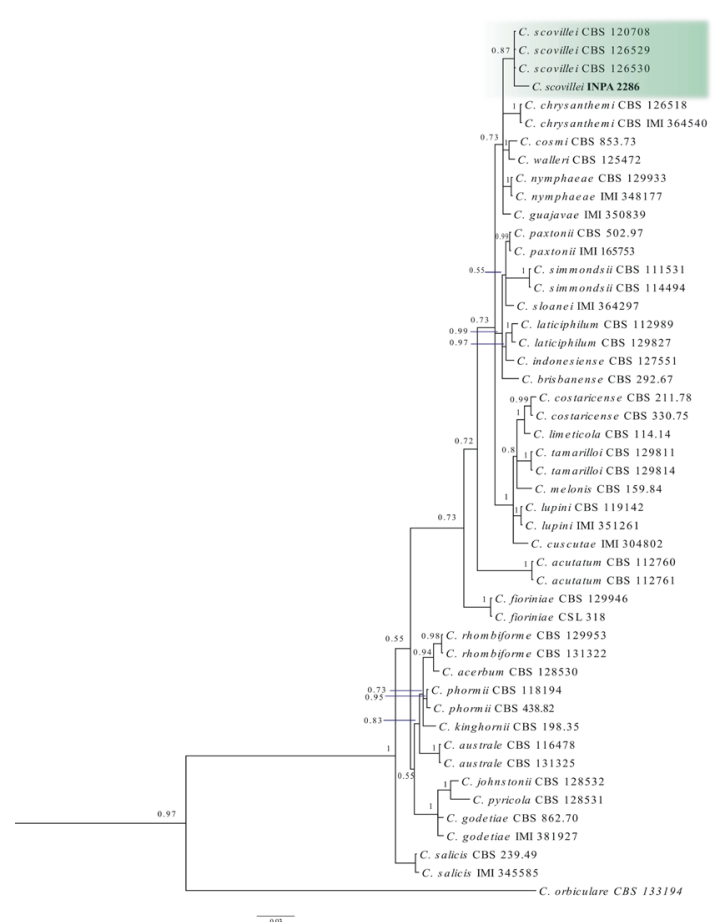

Figure 5. Phylogenetic tree generated by Bayesian inference upon a combinated ACT and GAPDH alignment sequences of Colletotrichum acutatum species complex among with the INPA 2286 isolate, highlighted. This tree is rooted with $C$. orbiculare. Relevant bootstrap values are shown ate the nodes.

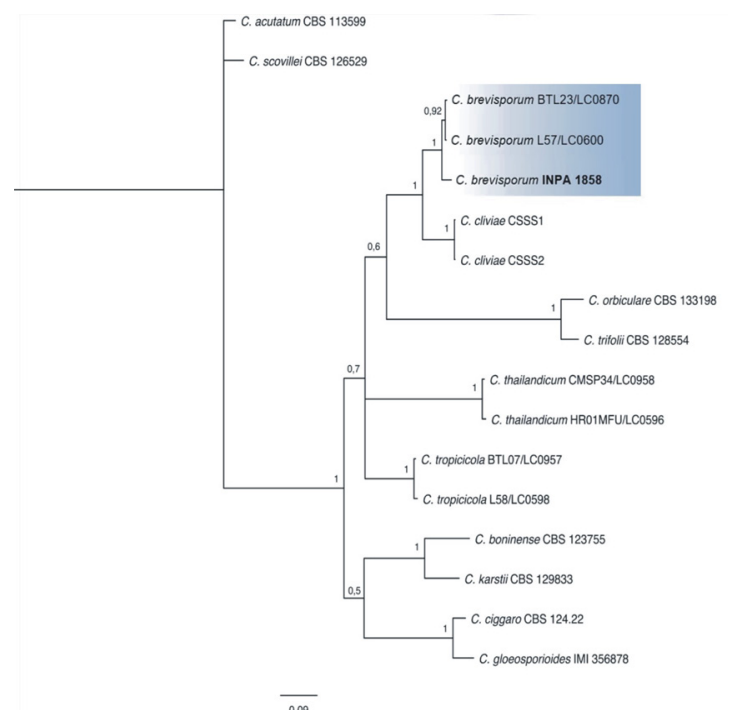

Figure 6. Phylogenetic tree generated by Bayesian inference upon a combinated ACT and GAPDH alignment sequences of Colletotrichum species including C. brevisporum among with the INPA 1858 isolate, highlighted. This tree is rooted with C. acutatum and C. scovillei. Relevant bootstrap values are shown ate the nodes. 
The combined dataset generated from Bayesian analysis shows similar topology with individual trees. In the phylogenetic trees based on combined dataset of ACT, GAPDH and SOD2 comprised 873 characters including alignment gaps, which showed that the isolate INPA 2066 is closely related to C. siamense H. Prihastuti, L. Cai \& K.D. Hyde. This tree was rooted to C. boninense (Figure 4). C. siamense is biologically and geographically diverse, found in many hosts across several tropical and subtropical regions, including Capsicum annuum in Thailand (Weir et al., 2012). Recently, Sharma et al. (2015) using multilocus analysis demonstrated that $C$. siamense are four distinct species forming the $C$. siamense species complex.

The analysis of the combined dataset of ACT and GAPDH showed that the isolate INPA 2286 formed a monophyletic clade supported (Bayesian posterior probability $=0.87$ ) with three strains of C. scovillei (Figure 5). And isolate INPA 1858 formed a monophyletic clade with high support (Bayesian posterior probability $=1$ ) with two isolates of $C$. brevisporum (Figure 6). The trees were rooted to $C$. orbiculare, and two species from each complex $(C$. acutatum and C. scovillei), respectively (Figures 5 and 6). Colletotrichum scovillei belongs to C. acutatum species complex and was associated with chilli in Indonesia and Thailand (Than et al. 2008; Weir et al., 2012).

In Brazil, the first report of anthracnose on pepper fruit $(C$. annuum L.) caused by $C$. scovillei was by Caires et al. (2014). Colletotrichum brevisporum, which is still not inserted in any Colletotrichum species complex has been reported in Neoregalia sp and Pandanus pigmaeus in Thaliand (Noireung et al., 2012). In Brazil, it has been notified the presence of this pathogen in papaya fruit (Vieira et al., 2013), chaoyte fruits (Bezerra et al., 2016) and chili pepper (Almeida et al., 2017).

In the present study, we showed that the molecular markers were able to distinguish the isolates of Colletotrichum studied through the different band profiles and was possible to differentiate isolates of the $C$. gloeosporioides and $C$. acutatum species complex. The phylogenetic analysis results confirmed the occurrence of $C$. siamense, $C$. scovillei and $C$. brevisporum causing anthracnose in $C$. chinense in the State of Amazonas.

This study represents the first report of the species $C$. siamense and $C$. scovillei in this host.

\section{Conflicts of interest}

The authors declare no conflict of interest.

\section{ACKNOWLEDGMENTS}

The authors thank Fundação de Amparo à Pesquisa do Estado do Amazonas (FAPEAM) and Coordenação de Aperfeiçoamento de Pessoal de Nível Superior (CAPES) for scholarships.

\section{REFERENCES}

Almeida LB, Matos KS, Assis LAG, Hanada RE, et al. (2017). First report of anthracnose of Capsicum chinense in Brazil caused by Colletotrichum brevisporum. Plant Dis. 101: 1035. https://doi.org/10.1094/PDIS-01-17-0099-PDN

Bezerra JP, Ferreira PV, Barbosa LF, Ramos-Sobrinho R, et al. (2016). First report of anthracnose on chayote fruits (Sechium edule) caused by Colletotrichum brevisporum. Plant Dis. 100: 217. https://doi.org/10.1094/PDIS-07-150793-PDN

Genetics and Molecular Research 16 (2): gmr16029601 
Caires NP, Pinho DB, Souza JSC, Silva MA, et al. (2014). First report of anthracnose on pepper fruit caused by Colletotrichum scovillei in Brazil. Plant Dis. 98: 1437. https://doi.org/10.1094/PDIS-04-14-0426-PDN

Damm U, Cannon PF, Woudenberg JHC, Johnston PR, et al. (2012a). The Colletotrichum boninense species complex. Stud. Mycol. 73: 1-36. https://doi.org/10.3114/sim0002

Damm U, Cannon PF, Woudenberg JH and Crous PW (2012b). The Colletotrichum acutatum species complex. Stud. Mycol. 73: 37-113. https://doi.org/10.3114/sim0010

Damm U, Cannon PF, Liu F, Barreto RW, et al. (2013). The Colletotrichum orbiculare species complex: Important pathogens of field crops and weeds. Fungal Divers. 61: 29-59. https://doi.org/10.1007/s13225-013-0255-4

Dias GB, Gomes VM, Pereira UZ, Ribeiro SFF, et al. (2013). Isolation, characterization and antifungal activity of proteinase inhibitors from Capsicum chinense Jacq. Seeds. Protein J. 32: 15-26. https://doi.org/10.1007/s10930-012-9456-Z

Doyle JJ and Doyle JL (1987). A rapid DNA isolation procedure for small quantities of fresh leaf tissue. Phytochem. Bull. 19: 11-15.

Liu B, Louws FJ, Sutton TB and Correll JC (2012). A rapid qualitative molecular method for the identification of Colletotrichum acutatum and C. gloeosporioides. Eur. J. Plant Pathol. 132: 593. https://doi.org/10.1007/s10658011-9904-1

Miller MA, Pfeiffer W and Schwartz T (2010). Creating the CIPRES Science Gateway for inference of large phylogenetic trees. In: Proceedings of the Gateway Computing Environments Workshop (GCE). New Orleans LA, USA.

Noireung P, Phoulivong S, Liu F, Cai L, et al. (2012). Novel species of Colletotrichum revealed by morphology and molecular analysis. Cryptogam., Mycol. 33: 347-362. https://doi.org/10.7872/crym.v33.iss3.2012.347

O'Connell RJ, Thon MR, Hacquard S, Amyotte SG, et al. (2012). Lifestyle transitions in plant pathogenic Colletotrichum fungi deciphered by genome and transcriptome analyses. Nat. Genet. 44: 1060-1065. https://doi.org/10.1038/ng.2372

Posada D and Buckley TR (2004). Model selection and model averaging in phylogenetics: advantages of akaike information criterion and Bayesian approaches over likelihood ratio tests. Syst. Biol. 53: 793-808.

Rambaut A (2012). FigTree version 1.4.0. Available at [http://tree.bio.ed.ac.uk/software/figtree].

Ratanacherdchai K, Wang H, Lin F and Soytong K (2010). ISSR for comparison of cross-inoculation potential of Colletotrichum capsici causing chilli anthracnose. Afr. J. Microbiol. Res. 4: 076-083.

Ronquist F, Teslenko M, van der Mark P, Ayres DL, et al. (2012). MrBayes 3.2: efficient Bayesian phylogenetic inference and model choice across a large model space. Syst. Biol. 61: 539-542. https://doi.org/10.1093/sysbio/sys029

Sharma G, Pinnaka AK and Shenoy BD (2015). Resolving the Colletotrichum siamense species complex using ApMat marker. Fungal Divers. 71: 247-264. https://doi.org/10.1007/s13225-014-0312-7

Tamura K, Stecher G, Peterson D, Filipski A, et al. (2013). MEGA6: Molecular Evolutionary Genetics Analysis version 6.0. Mol. Biol. Evol. 30: 2725-2729. https://doi.org/10.1093/molbev/mst197

Than PP, Jeewon R, Hyde KD, Pongsupasamit S, et al. (2008). Characterization and pathogenicity of Colletotrichum species associated with anthracnose on chili (Capsicum spp.) in Thailand. Plant Pathol. 57: 562-572. https://doi. org/10.1111/j.1365-3059.2007.01782.x

Vieira WAS, Nascimento RJ, Michereff SJ, Hyde KD, et al. (2013). First Report of Papaya Fruit Anthracnose Caused by Colletotrichum brevisporum in Brazil. Plant Dis. 97: 1659. https://doi.org/10.1094/PDIS-05-13-0520-PDN

Weir BS, Johnston PR and Damm U (2012). The Colletotrichum gloeosporioides species complex. Stud. Mycol. 73: 115180. https://doi.org/10.3114/sim0011

Genetics and Molecular Research 16 (2): gmr16029601 